\title{
Reconstructed bifurcation diagrams of CD4 cells and HIV-1 viral load dynamics under the effect of antiretroviral drugs
}

\author{
Krishnamurthy Kamalanand • Ponnuswamy Mannar Jawahar
}

Received: 12 July 2013/Revised: 3 November 2013/Accepted: 8 November 2013/Published online: 19 November 2013

(C) Springer-Verlag Wien 2013

\begin{abstract}
Nonlinear methods such as chaos theory and bifurcation diagrams are useful for analyzing complex biological and medical systems. In this work, the bifurcation diagrams of CD4 cells and viral load dynamics under antiretroviral therapy have been reconstructed from the measured time series using a nonlinear dynamic model. The bifurcation parameter of the system was identified and the bifurcation diagrams were constructed. Results demonstrate that the CD4 and HIV-1 viral load under the influence of antiretroviral drugs (zidovudine, lamivudine and ritonavir) exhibits bifurcation behavior in short parameter ranges. Further, it was observed that the antiretroviral therapy may fail at certain values of the bifurcation parameter $(\mu=7.769,7.883$ and 7.91), since there is a possibility of decrease in CD4 cell population at these values of the parameter.
\end{abstract}

Keywords HIV/AIDS - Bifurcation diagram ·

Chaos theory · CD4 cells · Viral load

\section{Introduction}

The measured time series representing the various dynamical aspects of infectious diseases can explain complex physiological and pathological conditions. The

\footnotetext{
K. Kamalanand $(\bowtie)$

Department of Instrumentation Engineering, MIT Campus, Anna University, Chennai 600 044, India

e-mail: kamalanand@mitindia.edu

P. M. Jawahar

Centre for University Industry Collaboration, Anna University, Chennai 600 025, India

e-mail:pmjmit@gmail.com
}

nonlinear analysis of the HIV system is highly useful for choosing appropriate modeling techniques and for designing suitable control strategies (de Souza 1999; Ho and Ling 2010). Further, mathematical models are widely utilized in medicine and biology for understanding the dynamics of biological processes (Gupta and Misra 2013; Sibanda and Pretorius 2013; Nounou et al. 2013).

There are several reports on the nonlinear and chaotic analysis of the HIV system without antiretroviral therapy. Ho and Ling (2010) have shown that the HIV system dynamics is sensitive to both the initial conditions and the system parameters. Kamalanand and Jawahar (2012a, b) have analyzed the predictability of the HIV system using chaotic measures such as the Hurst exponents. The authors have further used nonlinear estimation techniques such as particle swarm optimization and jumping frogs optimization for estimation of the HIV system parameters (Kamalanand and Jawahar 2012a, b; 2013a, b).

Al-Sheikh et al. (2011) performed stability analysis on a nonlinear mathematical model which analyzes the spread and control of HIV. Bortz and Nelson (2004) performed a formal sensitivity analysis on a nonlinear model of HIV infection dynamics. However, the reports on chaotic analysis on HIV system under the influence of antiretroviral drugs are very limited.

The bifurcations in dynamical systems are the qualitative changes in dynamics of the system. The parameter values at which they occur are called bifurcation points. The bifurcation diagram provides models of instabilities in the dynamical system (Strogatz 1994) and is an important tool in chaos theory for understanding the periodic and chaotic properties of a nonlinear system. Further, the bifurcation diagrams are useful in analyzing the steadystate solutions of this system for variations in system parameters. The bifurcation diagrams are important for 
obtaining the area of dynamic system parameter for which the system behavior is chaotic (Bagarinao et al. 2000). Recently, bifurcation diagrams have been utilized in a variety of fields for the purpose of analysis (Lou et al. 2013; de Back et al. 2013; Kamalanand and Jawahar 2013a, b).

The objective of this work is to reconstruct the bifurcation diagrams of CD4 cell population and viral load dynamics of the HIV system under the influence of antiretroviral drugs_zidovudine, lamivudine and ritonavir.

\section{Methodology}

In this work, the bifurcation diagrams of CD4 cells and viral load dynamics under therapy have been reconstructed from the measured time series using the method proposed by Bagarinao et al. (2000). The ACTG 315 longitudinal data $(N=46)$ consisting of CD4 cell count and HIV-1 viral load under the drug combination consisting of zidovudine (AZT), lamivudine (3TC) and the protease inhibitor ritonavir (Connick et al. 2000; Lederman et al. 1998; Liang et al. 2003; Wu and Ding 1999; Wu et al. 1999; Wu and Liang 2004) has been utilized for this study. A dynamic polynomial model of the form:

$$
\begin{aligned}
x(t)= & a_{0}+a_{1} x(t-1)+a_{2} x(t-1)^{2}+a_{3} x(t-1)^{3} \\
& +a_{4} x(t-1)^{4}
\end{aligned}
$$

was used for describing the dynamics of CD4 cell count and HIV-1 viral load, where, $a_{i}=\left[a_{0}, a_{1}, a_{2}, a_{3}, a_{4}\right]$ is the set of parameters of the model and $x(t)$ is the measured time series. The bifurcation parameter $(\mu)$ from the set of model parameters was selected as the parameter exhibiting maximum variance. Further, the bifurcation diagrams of CD4 cells and viral load dynamics were reconstructed using $\mu$ as the effective bifurcation parameter. The algorithm (Houfek et al. 2008) for constructing the bifurcation diagrams is presented:

Algorithm 1: Reconstruction of bifurcation diagrams from measured time series

Step 1: Develop dynamic nonlinear models of the form $x(n)=f(x(n-1))$, parameterized by a set of parameters, from the measured time series.

Step 2: Select the bifurcation parameter from the parameter set, as the parameter exhibiting maximum variance, and determine the interval of the parameter change.

Step 3: Perform the solution of the model which describes the system under consideration, depending of the particular value of bifurcation parameter.

Step 4: Introduce small change in the value of bifurcation parameter.
Table a continued

Algorithm 1: Reconstruction of bifurcation diagrams from measured time series

Step 5: Repeat steps 3 and 4 until the full interval of bifurcation parameter is fulfilled.

Step 6: Draw bifurcation diagram where each partial calculation corresponds to a single point.

\section{Results and discussion}

Figure 1 shows the reconstructed bifurcation diagram of CD4 cell dynamics under the influence of antiretroviral drugs. It is seen that the CD4 cells exhibit bifurcation behavior in the short parameter range of 7.5-8.1. It is found that CD4 cells exhibit pitchfork bifurcations at $\mu=7.769$, 7.883 and 7.91 . Hence, it appears that there is a possibility of failure in antiretroviral therapy beyond the bifurcation points, since by increasing these values of the system parameters, the CD4 cell count may decrease or increase.

Figure 2 shows the reconstructed bifurcation diagram of HIV-1 viral load dynamics under the influence of antiretroviral drugs. It appears that the viral load also exhibits several bifurcations as the parameter value decreases in the range of $0.7-0.9$. It is seen that the HIV-1 viral load exhibits pitchfork bifurcations at $\mu=0.843,0.823$ and 0.753 .

Further, it appears that both the CD4 cell population and HIV-1 viral load exhibit chaotic and bifurcation behavior in the considered range of parameters, under the influence of antiretroviral drugs such as zidovudine, lamivudine and ritonavir and any change in the value of the bifurcation parameter results in instability of the therapy.

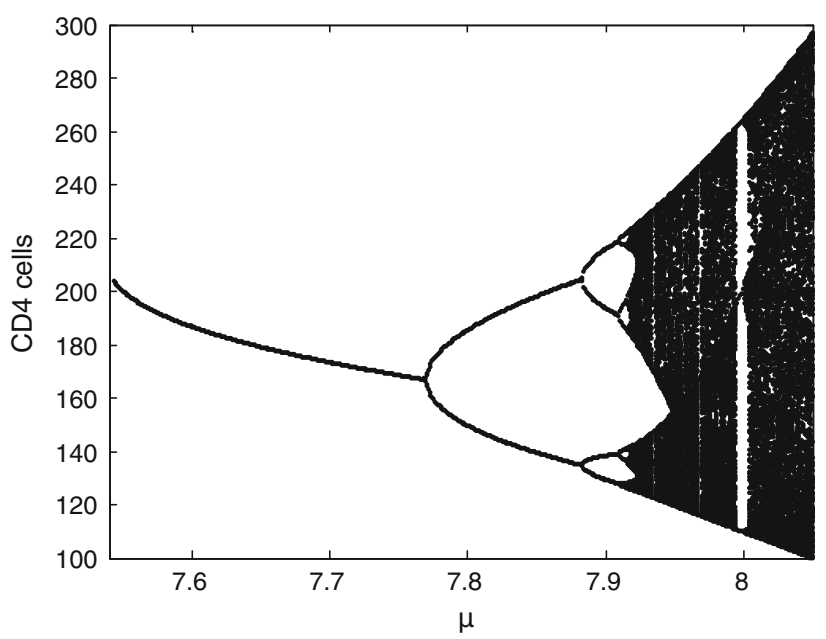

Fig. 1 Reconstructed bifurcation diagram of CD4 cell dynamics under the influence of antiretroviral drugs 


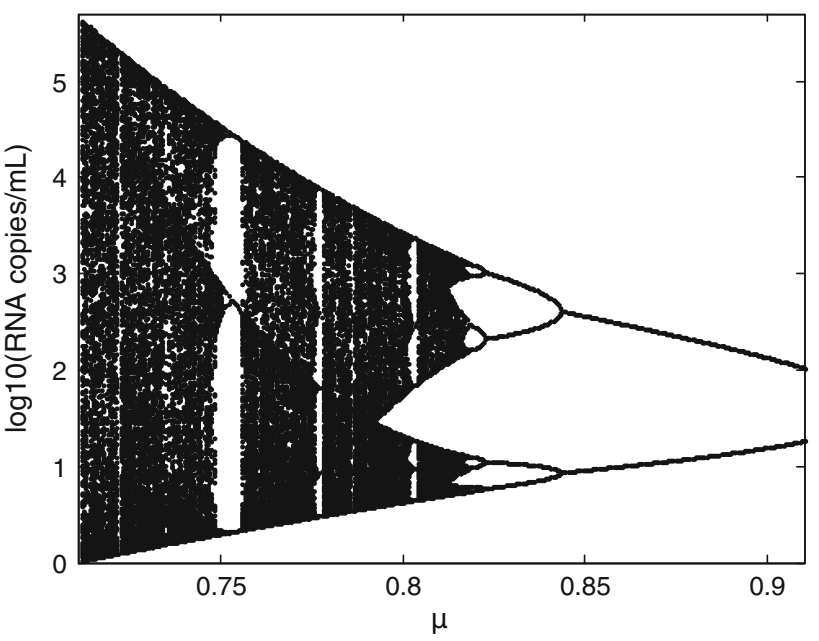

Fig. 2 Reconstructed bifurcation diagram of HIV-1 viral RNA dynamics under the influence of antiretroviral drugs

\section{Conclusions}

The bifurcation diagram is an important tool for analyzing the periodic and chaotic properties of a nonlinear system. In this work, the bifurcation diagrams of CD4 cells and viral load dynamics under the influence of antiretroviral drugs have been reconstructed using a dynamic nonlinear model, from the measured time series.

Results demonstrate that the CD4 and HIV-1 viral load under the influence of antiretroviral drugs zidovudine, lamivudine and ritonavir exhibit bifurcation behavior and is chaotic in short parameter ranges. Further, it was observed that the antiretroviral therapy may fail at particular values of the bifurcation parameter. It appears that this study is of high clinical relevance since it explains the effect of antiretroviral drugs on the chaotic and bifurcation behavior of the HIV system.

\section{References}

Al-Sheikh S, Musali F, Alsolami M (2011) Stability analysis of an HIV/AIDS epidemic model with screening. Int Math Forum 6:3251-3273

Bagarinao E, Pakdaman K, Nomura T, Sato S (2000) Reconstructing bifurcation diagrams of dynamical systems using measured time series. Meth Info Med 39:146-149

Bortz DM, Nelson PW (2004) Sensitivity analysis of a nonlinear lumped parameter model of HIV infection dynamics. Bull Math Biol 66:1009-1026

Connick E, Lederman MM, Kotzin BL, Spritzler J, Kuritzkes DR, St Clair M, Sevin AD, Fox L, Chiozzi MH, Leonard JM, Rousseau F, D'Arc Roe J, Martinez A, Kessler H, Landay A (2000) Immune reconstitution in the first year of potent antiretroviral therapy and its relationship to virologic response. J Infect Dis 181:358-363 de Back W, Zimm R, Brusch L (2013) Transdifferentiation of pancreatic cells by loss of contact-mediated signaling. BMC Syst Biol 7(77):2-11

de Souza FMC (1999) Modeling the dynamics of HIV-1 and CD4 and CD8 lymphocytes. IEEE Eng Med Biol 18:21-24

Gupta MK, Misra K (2013) Modeling and simulation analysis of propyl-thiouracil (PTU), an anti-thyroid drug on thyroid peroxidase (TPO), thyroid stimulating hormone receptor (TSHR), and sodium iodide (NIS) symporter based on systems biology approach. Network Modeling Analysis in Health Informatics and Bioinformatics 2(1):45-57

Ho CYF, Ling BWK (2010) Initiation of HIV therapy. Int J Bifurcat Chaos 20:1279-1292

Houfek L, Kratochvil C, Houfek M (2008) Bifurcation and chaos in drive systems. Eng Mech 15:401-410

Kamalanand K, Jawahar PM (2012a) Predictability analysis on HIV/ AIDS system using hurst exponents. Int $\mathrm{J}$ Med Biol Sci 6:301-305

Kamalanand K, Jawahar PM (2012b) Coupled jumping frogs/particle swarm optimization for estimating the parameters of three dimensional HIV model. BMC Infect Dis 12(Suppl 1):82

Kamalanand K, Jawahar PM (2013a) Particle swarm optimization based estimation of HIV-1 viral load in resource limited settings. Afr J Microbiol Res 7:2297-2304

Kamalanand K, Jawahar PM (2013b) A mathematical exploration into manipulation and control of a bifurcative dreaming process. Int $\mathbf{J}$ Dream Res 6(2):40-43

Lederman MM, Connick E, Landay A, Kuritzkes DR, Spritzler J, St Clair M, Kotzin BL, Fox L, Chiozzi MH, Leonard JM, Rousseau F, Wade M, Roe JD, Martinez A, Kessler H (1998) Immunologic responses associated with 12 weeks of combination antiretroviral therapy consisting of zidovudine, lamivudine and ritonavir: results of AIDS Clinical Trials Group Protocol 315. J Infect Dis 178:70-79

Liang H, Wu H, Carroll RJ (2003) The relationship between virologic responses in AIDS clinical research using mixed-effects varyingcoefficient models with measurement error. Biostatistics 4:297-312

Lou IC, Zhao Y, Wu Y, Ricci PF (2013) Systems cancer biology and the controlling mechanisms for the J-shaped cancer dose response: towards relaxing the LNT hypothesis. Dose Response 11(3):301-318

Nounou MN, Nounou HN, Mansouri M (2013) Model-based and model-free filtering of genomic data. Netw Model Anal Health Inform Bioinform 2(3):109-121

Sibanda W, Pretorius P (2013) Comparative study of the application of central composite face-centred (CCF) and Box-Behnken designs (BBD) to study the effect of demographic characteristics on HIV risk in South Africa. Netw Model Anal Health Inform Bioinform 2(3):137-146

Strogatz SH (1994) Nonlinear dynamics and chaos. Perseus, London

Wu H, Ding A (1999) Population HIV-1 dynamics in vivo: applicable models and inferential tools for virological data from AIDS clinical trials. Biometrics 55:410-418

Wu H, Liang H (2004) Backfitting random varying-coefficient models with time-dependent smoothing covariates. Scan J Statist 31:3-19

Wu H, Kuritzkes DR, McClernon DR, Kessler H, Connick E, Landay A, Spear G, Heath-Chiozzi M, Rousseau F, Fox L, Spritzler J, Leonard JM, Lederman MM (1999) Characterization of viral dynamics in human immunodeficiency virus type 1-infected patients treated with combination antiretroviral therapy: relationships to host factors, cellular restoration and virological endpoints. J Infect Dis 179:799-807 